\section{CMOS tunable linear current divider}

\author{
C.H.J. Mensink and B. Nauta
}

Indexing terms: CMOS integrated circuits, Dividing circuits

A CMOS current divider is proposed which can handle an input current as large as the bias current, i.e. the modulation depth can be $100 \%$. The performance of the circuit is nearly independent of transistor characteristics. Therefore the circuit is well applicable for designs in modern submicron processes. The measured divider can be tuned over more than a factor 2 .

Introduction: On-chip integrated circuits often need to be tunable to correct for process or temperature variations. However, unpredictable or unknown device characteristics make it hard to implement tunable linear circuits. In modern submicron CMOS technologies, the transistor characteristics can deviate significantly from the ideal behaviour. In this Letter a continuously tunable current divider is presented which is rather insensitive for MOS transistor qualities. Furthermore, the amplitude of the input current may be equal to the bias current. The divider is well suitable for analogue video circuits.

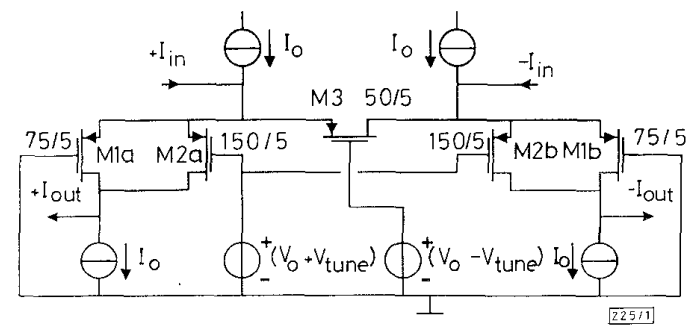

Fig. 1 Schematic diagram of tunable linear current divider

Circuit description: The current divider, shown in Fig. 1, consists of five $P$-MOS transistors and has a differential input and output. The backgates of these transistors are connected to the same constant voltage, e.g. the supply voltage. The voltage $V_{0}$ is $\sim 0.5\left|V_{T}\right|$ where $V_{T}$ is the threshold voltage. The value of the tune voltage $V_{\text {tune }}$ is between $-V_{0}$ and $V_{0 j}$. The circuit has three special operating points where the transfer is almost linear, namely for $V_{\text {tume }}$ it equals $-V_{0}, V_{0}$ and close to zero. These points are discussed below.

(i) If $V_{\text {tune }} \leq-V_{0}$, transistor $\mathrm{M} 2$ is in parallel with $\mathrm{M} 1$, and M3 does not conduct. The input current flows directly to the output without any distortion. The current gain is exactly 1 .

(ii) If $V_{\text {tune }}$ equals $V_{0}, \mathrm{M} 2$ is turned off. The gate of $\mathrm{M} 3$ is grounded, and M3 is in series with M1. It has been proven by Bult and Geelen [1] that a current fed to two transistors in series with the same gate and backgate voltages splits linearly in a ratio determined by the aspect ratios of the MOS transistors, independent of the operation mode (the division technique in [1] is used in a digitally tuned circuit, in contrast to the proposed circuit here, where the tuning is analogue). If $V_{t u m}$ is equal to $V_{0}$, the situation is similar to that described in [1]. The current division between M1 and M3 is inherently linear. Only a fraction of the input current, determined by the aspect ratios of $\mathrm{Ml}$ and M3, flows to the output. Note that the tuning does not affect the bias current of the circuit.

(iii) Besides both extremes, the transfer will also be linear if $V_{\text {tune }}$ is approximately zero. For $V_{\text {tume }}=0$, the current division between M2 and M3 is perfectly linear. However, the current through M1 is not a linear fraction of the current through M2 and M3. The transfer from input current to output current is not perfectly linear. However, it appears that, for a tune voltage slightly different from zero, overall compensation will occur, resulting in a low distortion operating point.

The transistor M2 is multifunctional. First, if $V_{\text {tume }}$ is slightly larger than $-V_{0}$, the current division is not linear. The distortion is mainly determined by the operation modes of the devices. Since M3 is in moderate inversion, it has a rather nonlinear drain-source resistance. The transistor M2 is needed to keep the voltage swing across M3 small, resulting in a small current through M3. Secondly, due to $\mathrm{M} 2$, an extra low distortion operating point is introduced, as described above. Thirdly, thanks to turning off M2 when $V_{\text {tume }}$ meets $V_{0}$, the output noise current is reduced since the total impedance is increased. The noise current is determined by the series-connection of M1 and M3, disregarding the noise of the bias current.

Measurement results: The circuit has been realised in a $0.5 \mu \mathrm{m}$ CMOS process. The $W / L$ dimensions, in $\mu \mathrm{m} / \mu \mathrm{m}$, are indicated in Fig. 1. The bias current $I_{0}$ is equal to $75 \mu \mathrm{A}, V_{0}$ is $0.5 \mathrm{~V}$ and the threshold voltage is $0.9 \mathrm{~V}$. The substrate of the $P$-MOS transistors is connected to a $+3.3 \mathrm{~V}$ supply voltage.

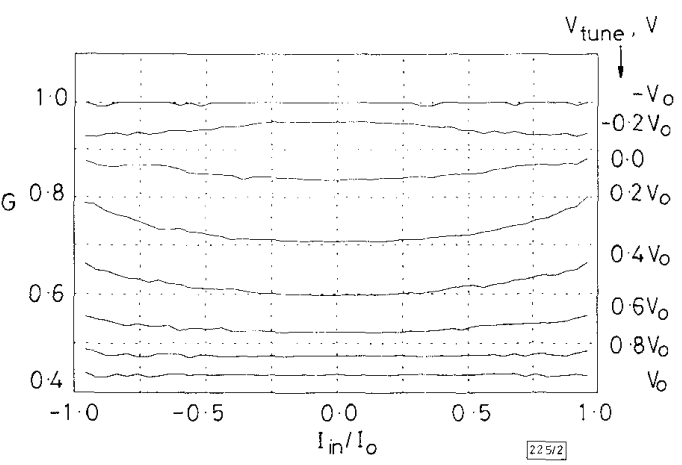

Fig. 2 Gain $G=d I_{\text {out }} / d I_{\text {in }}$ for $I_{0}=75 \mu \mathrm{A}$ and $V_{0}=0.5 \mathrm{~V}$

The gain $G$ of the circuit is defined by the derivative of the output current to the input current, $d I_{o u t} / d I_{\text {in }}$. In Fig. 2 the measured gain is depicted for several values of the tune voltage. The input current varies from $-75 \mu \mathrm{A}$ to $75 \mu \mathrm{A}$, which means that the modulation depth, defined by the ratio of the input current and the bias current $I_{i n} / I_{0}$, varies between $-100 \%$ and $100 \%$. The gain does not roll off for large values of the modulation depth. This relies on two facts. First, the current division technique as described in [1] is valid in strong inversion as well as in moderate and weak inversion. Secondly, when M3 conducts, the eurrent can flow from the left to the right branch and vice versa. In that case, the current through M1 and M2 is never zero even when the input current equals plus or minus $I_{0}$. One can clearly see that the linearity for the extremes, where $V_{\text {tume }}$ equals $-V_{0}$ and $V_{0}$, is superior. Furthermore, when the tune voltage varies from $-0.2 V_{0}$ to $0 \mathrm{~V}$, the gain changes from a convex to a concave shape. This implies that the gain must be linear for a particular tune voltage. The tuning range is determined by the aspect ratio of $\mathrm{M} 1$ and twice the aspect ratio of M3, due to the balancing. The minimum gain is equal to $G_{\min }=$ $75 /(75+2 \times 50)=0.43$

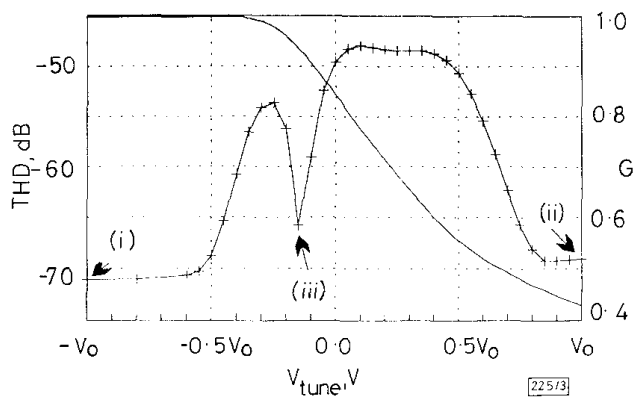

Fig. $3 T H D$ and gain, for $f=1 \mathrm{kHz}, I_{0}=75 \mu \mathrm{A}, V_{0}=0.5 \mathrm{~V}$, and $I_{\text {ininar }}=$ $50 \mu \mathrm{A}$

- + - THD, — G

The THD figures are given in Fig. 3. The differential input current was generated by an on-chip $V / I$ converter consisting of a poly-resistor and two op-amps. Again the bias current $I_{0}$ is $75 \mu \mathrm{A}$. The input current has a frequency of $1 \mathrm{kHz}$ and an amplitude of $50 \mu \mathrm{A}$, so the maximum modulation depth is $66 \%$. The distortion due to the $V / I$ converter is $-71 \mathrm{~dB}$, which limits the distortion figure at point (i) (Fig. 3). For values of $V_{\text {tune }}$ between the points (ii) 
and (iii), the distortion is mainly determined by M3 since it operates in moderate inversion. Owing to the wide transistor M2, the current through M3 remains small, resulting in low distortion figures. The distortion has a local minimum at a $V_{\text {tare }}$ value of $-0.15 V_{0}$ (point (iii)), which is close to a $V_{\text {tuse }}$ value of $0 \mathrm{~V}$, where the division between M2 and M3 is linear. For values of $V_{\text {uимe }}$ between the points (iii) and (ii), the distortion is chiefly induced by M2, which is somewhere in moderate inversion. The voltage variation at input nodes causes a nonlinear current in M2. Owing to the drain-source resistance of M3, the voltage variation is reduced leading to a fairly linear transfer. If $V_{\text {tune }}$ equals $V_{0}$, the circuit is not perfectly linear due to second-order effects at the drain of $M 1$ [1]. Measurement results of the same circuit with short channel devices, i.e. $0.5 \mu \mathrm{m}$, show that the distortion at this point is $\sim 6 \mathrm{~dB}$ larger, but barely affects the distortion figures for lower values of $V_{\text {tune }}$.

Conclusions: A compact tunable current divider has been presented with a low sensitivity for the MOS characteristics and is very suitable for future processes. The current modulation depth can go up to a $100 \%$. The current divider has a worst-case distortion of $-48 \mathrm{~dB}$ for a modulation of depth $66 \%$. The tuning mechanism does not affect the bias current of the circuit, which is generally an advantage for biasing peripherals.

Acknowledgments: This work is supported by Philips Research Laboratories Eindhoven, The Netherlands. The authors would like to thank $\mathrm{H}$. Wallinga and R. F. Wassenaar of the University of Twente for their contribution.

(c) IEE 1996

Electronics Letters Onlie No: 19960620

1 February 1996

C.H.J. Mensink (Mesa Research Institute, University of Twente Department of Electrical Engineering, PO Box 217, 7500 AE Enschede, The Netherlands)

B. Nauta (Philips Research Laboratories, Prof. Holstlaan 4, 5656 A A Eindhoven, The Netherlands)

\section{References}

1 BULT, K., and GEELEN, G.J.G.M.: 'An inherently linear and compact MOST-only current division technique', IEEE J. Solid-State Circuits, 1992, 27, (12), pp. 1730-1735

\section{Numerical simulation of local charging during plasma etching of a dielectric material}

\author{
A. Shibkov, M.K. Abatchev, H.K. Kang and M.Y. Lee
}

Indexing terms: Plasma, Vltra-large-scale integration etching

Local pattern charging during plasma etching of a dielectric material is investigated by means of two-dimensional MonteCarlo simulation. Detailed surface charge and potential distributions are presented and analysed. The differential pattern charging is shown to induce an electric field which is strong enough to cause considerable ion energy reduction and etched profile distortion.

Introduction: The plasma etching process has played a key role in integrated circuit manufacture for many years, and it becomes even more important nowadays as we enter the era of ultra-largescale integration. However, the etching rate and profile distortions of the small features were found to depend on the structure geometry and process conditions. The etching rate dependence on the feature size results in the necessity to do some, sometimes significant, overetching to achieve equal depths of the features with different sizes. This in turn increases the cost of manufacturing and makes the chips vulnerable to damage due to overetching or failure to clear. Deviations from the desired profile can also lead to considerable yield reduction. As a result the dependence of the etching rate and profile on both the feature size and the process conditions has been widely discussed in the literature [1 -9] and several attempts have been made to develop the models of the etching process $[3-5,8,9]$. In the present Letter we discuss the influence of local charging effects on the dielectric material etching process. While the important role of local charge buildup during the plasma etching of an insulator has been mentioned several times $[3-6,8]$, we are aware of only one attempt at numerical simulation of local pattern charging during dielectric material etching, which was made by Arnold et al. [4]. Despite their importance, results presented in [4] were difficult to interpret because of high numerical noise caused by rough discretisation of the angle and velocity distributions

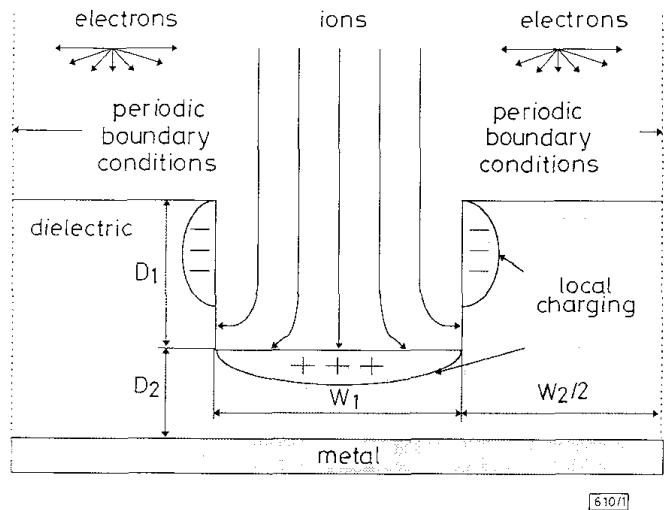

Fig. 1 Schematic diagram of etching geometry considered in this Letter

Numerical approach: In this Letter we present the results of twodimensional simulation of the local charge buildup caused by plasma etching of a trench in the dielectric material. The MonteCarlo procedure was employed in which particle trajectories were calculated in a self-consistent electric field. The schematic diagram of the structure considered in this Letter is shown in Fig. 1. Periodic boundary conditions were used at the right and left boundaries of the modelling region. This is consistent with the geometry of some typical submicron structures, for example, memory chips. The potential of the metallic substrate was set to zero. Particles were generated at the top boundary of the simulation region. We took into consideration only electrons and positively charged ions. Electrons had an isotropic angle and Maxwellian velocity distribution. Ions were considered to have $\delta$-function-like energy distribution and velocity direction normal to the surface. In our simulation we assumed the dielectric material to be perfectly insulating. We did not take into account any kind of possible charge transfer by means of the surface or bulk current or surface discharge because details of these processes were not clear. In other words, the particles impinging the surface of a dielectric were considered to remain at the point where they hit the surface. Although our model did not include any material-specific parameters, in the following discussion of our results we refer to the case of $\mathrm{SiO}_{2}$ etching as one of the very important processes in semiconductor manufacturing.

Results and discussion: To investigate the effects of differential charging we simulated potential and surface charge distributions in the structure depicted in Fig. 1. For our simulation we have chosen the trench depth $D_{1}=1.2 \mu \mathrm{m}$, width $W_{1}=0.3 \mu \mathrm{m}$ and the thickness of an insulator at the trench bottom, $D_{2}=0.4 \mu \mathrm{m}$; the distance between the neighbouring trenches, $W_{2}$, was set to $0.3 \mu \mathrm{m}$. The dielectric constant of the insulator was chosen to be equal to 1. Ions were considered to be $10^{4}$ times heavier than electrons, while the absolute value of the ion charge was equal to that of an electron. Electron thermal energy and ion energy were equal to $3 \mathrm{eV}$ and $100 \mathrm{eV}$, respectively. The distance between the surface of the dielectric material and the top boundary of the simulation region was set to $3 \mu \mathrm{m}$. At this distance the electric field can already be considered as one-dimensional in the case of the simulated structure geometry [5], so that such truncation of the simulation region is justified. Calculated distributions of the potential and charge of the trench surface are shown in Figs. 2 and 3 , respectively. Fig. 3 shows that the bottom of the trench is charged 